International Journal of Wireless \& Mobile Networks (IJWMN) Vol. 4, No. 4, August 2012

\title{
SIMULATION STUDIES ON AN ENERGY EFFICIENT Multipath Routing Protocol Using DireCtionAL ANTENNAS FOR MANETS
}

\author{
Sandhya Chilukuri ${ }^{1}$, Rinki Sharma ${ }^{2}$, Deepali. R. Borade ${ }^{3}$ and Govind R. \\ $\mathrm{Kadambi}^{4}$ \\ Department of Computer Engineering, M. S. Ramaiah School of Advanced Studies, \\ Bengaluru, India \\ reachsandhyach@gmail.com; govind@msrsas.org; rinki@msrsas.org; \\ borade.deepali21@gmail.com
}

\begin{abstract}
The paper proposes the development of an energy efficient multipath routing protocol with directional antenna for MANET as an optimization task as well as a multidisciplinary entity. A comprehensive analysis to link all the multi-disciplinary viewpoints involved in the development of desired multipath routing protocol with requisite technical details is presented in this paper. A simple and elegant mathematical formulation for the analysis of relative improvement of the performance metrics of ad-hoc networks with omnidirectional and directional antenna is presented. Through extensive numerical simulations, the multi-dimensional desirable performance attributes of wireless link such as improved range, improved RSS, reduced RF transmit power and consequent reduced consumption of battery power have been analyzed keeping the directional gain of the antenna as a variable parameter. Development or modification of a protocol with concurrent focus on multipath routing with optimization of the battery energy is a significant step to increase the life time of MANET without recharging. Selection of the energy efficient path amongst several alternative ones is of paramount significance in the evaluation of overall performance of MANET system. A formulation to compute the required Battery Energy taking into account the data pertaining to the power efficiency of the associated transceiver design as well as the specified link performance parameters is also discussed. Through a case study involving the specifications of a typical transceiver operating in the $2.4 \mathrm{GHz}$ band, the desirable impact of higher gain of a directional antenna in the reduction of RF transmitter power is illustrated. The consequential reduced battery power consumption while still retaining the specified performance parameters of the adhoc network like range and Received Signal Strength (RSS) is also demonstrated. This paper also addresses the importance of alignment of beam peaks of directional antennas of a link and the quantification of additional RF power in lieu of Beam Pointing Angle (BPA) error in ad-hoc network. The profile of improved range with directional gain as an independent variable exhibits much sharper feature than an exponential function. The relationship between improvement in the RSS and higher directional gain bears linear characteristics and typical results reveal that for a $d B$ increase in gain ratio, the corresponding improvement in RSS is $2 \mathrm{dBm}$. Results of Proposed Routing protocol simulations with built in hop count reduction feature reveal energy saving of $62.11 \%$ for a typical MANET scenario of 25 nodes, $5 \mathrm{MB}$ data and data rate of $40 \mathrm{Kbps}$.
\end{abstract}

\section{Keywords}

Ad-Hoc Network, Mobile Ad-Hoc Network, Energy Efficient, Directional Antenna, Routing Protocol,

\section{INTRODUCTION}

Multi Hop Wireless Network has already emerged and continues to be a topic of hot research pursuit. An ad-hoc network is established with multiple mobile nodes, coming in range of each other and exchanging data without an access point. The ability of participating nodes to move around gives rise to Mobile Ad hoc Networks (MANETs). Such networks are very useful for

DOI : 10.5121/ijwmn.2012.4409 
military applications, emergency and rescue operations, health care, home networking and other commercial and educational applications. In a MANET, nodes are highly interdependent for exchange of information with each other. The nodes must cooperate to routing and other services, making it crucial to maximize the network lifetime. The participating nodes in a MANET (laptops, PDA's and sensors) have limited battery power; therefore energy efficient communication approach is critical for the longevity and efficiency of the network. To support energy-efficient communication in an Ad-hoc network, it is important to apply energy-efficient design at multiple layers of the network protocol stack. To realize an energy efficient ad-hoc network is to minimize the RF transmit power for conservation of battery power. In view of this, this paper emphasizes the application of directional antenna to realize the reduction in transmitter power and yet maintaining the desired link performance or network performance.

In this paper, we concentrate on the physical layer techniques used for energy-efficient communication in an attempt to illustrate the enhanced performance of Ad-hoc networks with directional antenna. Analysis to link the significant design parameters of directional antenna to wireless link performance is of practical significance to appreciate the system design considerations of ad-hoc networks. Through simple and elegant mathematical formulation, relative improvement of the performance metrics of ad-hoc network is analyzed when high gain directional antenna is replaced with conventional low gain omni directional antenna. Resource utilization particularly that of electric power of battery and the associated energy is of paramount importance since it has significant say in the outage condition of a wireless link or network. Through a case study involving the specifications of a typical transceiver operating in the $2.4 \mathrm{GHz}$ band, the desirable impact of higher gain of a directional antenna in the reduction of RF transmitter power is illustrated. This in turn results in the reduced consumption of battery power, while still retaining the specified performance parameters of the ad-hoc network such as range and Received Signal Strength (RSS). Although, directional antenna offers potentially many desirable performance improvement attributes, it is also associated with additional constraint or requirement of precise or accurate alignment of the directive or main beam of the Transmitter and Receiver. The mismatch of angular alignment of the Transmitter and Receiver results in Beam Pointing Angle (BPA) error, which leads to the degradation in the range. If one desires to regain the desirable ideal network performance despite the presence of BPA error, it would call for additional RF Transmitter power ultimately culminating in the consumption of extra power of the battery that support the RF operation. This paper also facilitates the quantification of additional RF power in lieu of BPA error in ad-hoc network.

Development or Modification of a protocol with concurrent focus on multipath routing with optimization of the battery energy is a significant step to increase the life time of MANETs without recharging. Selection of the energy efficient path amongst several alternative ones is of paramount significance in the evaluation of overall performance of MANET system. The desired protocol development is an optimization exercise involving multidisciplinary aspects of Antenna Engineering, Microwave Link Budget to treat intermediate wireless links, Algorithms to determine the most efficient path for reliable connectivity between the source and destination nodes, comprehensive relation between the RF power and the pertinent Battery Power of the transmit nodes involving conventional electrical power engineering basics as well as the choice and optimization of route metrics to arrive at decision logic for the path selection.

\section{COMPREHENSIVE SYSTEM VIEW OF MANET}

Figure 1 depicts a comprehensive System View of energy efficient multipath MANET routing protocol. Antenna or microwave link budget includes the concept of gain, link data, battery energy, range, RF power, RSS which are interrelated with each other. A perfect combination of them will help in conserve energy while routing. 


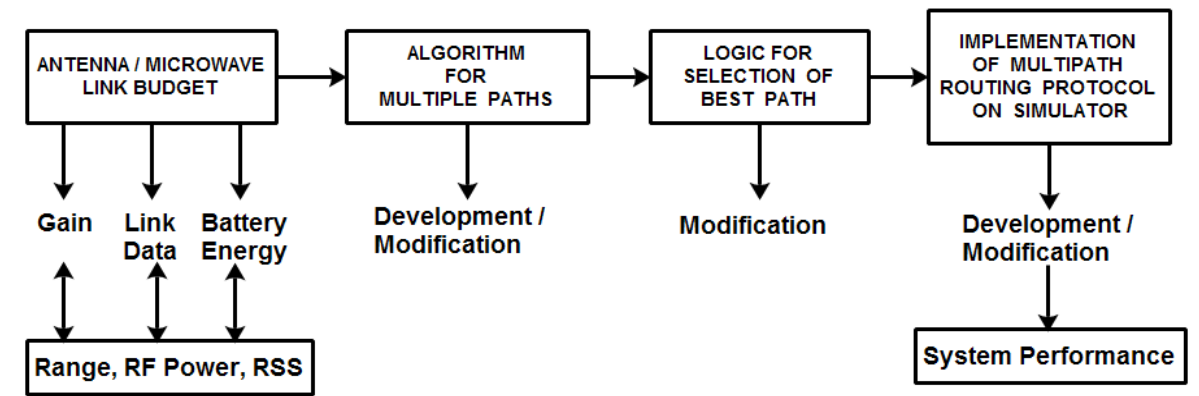

Figure 1: Comprehensive System View of Energy Efficient Multipath MANET Routing

Protocol

From the system design perspective, this paper addresses basically two broad system entities namely antenna and its relevance to energy efficiency considerations in MANET.

\section{Directional AnTenna in Mobile Ad Hoc Networks (MANETs)}

A representative generic MANET with three mobile nodes is shown in Figure 2 with the dotted circles, implying the communication range of each node. Each of the nodes is assumed to be design configured with a RF transceiver as their network interface. If Node A wishes to communicate with Node $\mathrm{C}$ and if Node $\mathrm{C}$ is not in communication range of Node A, Node $\mathrm{B}$ will serve as an intermediate node resulting in 2 hop communication.

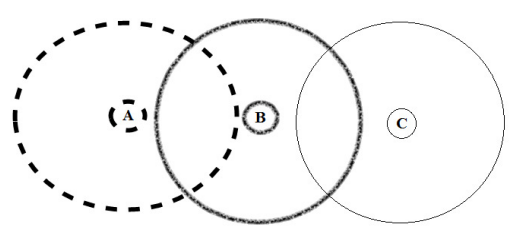

Figure 2. Multi Hop Communication in MANET

A transceiver of a MANET is configured with an antenna which will serve as its RF front end to establish a wireless communication link with its intermediate (participating) nodes. Basically, the antenna can be of two types namely omnidirectional and directional. Figure 3 shows the difference between radiation patterns of directional and omnidirectional antenna. It can be seen that the radiation pattern of omnidirectional antenna exhibits uniform angular distribution while the radiation pattern of directional antenna is concentrated in the preferred angle (direction) of communication. In Figure 3, S and D denote the source and destination nodes respectively. As illustrated in Figure 3, the communication range exhibited by directional antenna will be higher than an omnidirectional antenna. In view of this, it is implicit that the transceivers of nodes in Figure 2 are associated with omnidirectional antennas. In the past, omnidirectional antennas did find extensive applicability in MANET system design. With the rapid advancement of antenna technology conjunctured with miniaturization in size and its proven system utility in subscriber end of cellular communication, increasingly one finds rapid progression of directional antenna in ad-hoc networks/MANETs. The characterization of directional antenna to realize performance improvements in ad-hoc networks/MANETs wherein mobility is a key factor causing potentially dynamic and vast variations in communication range, constitutes the core theme of this paper. 

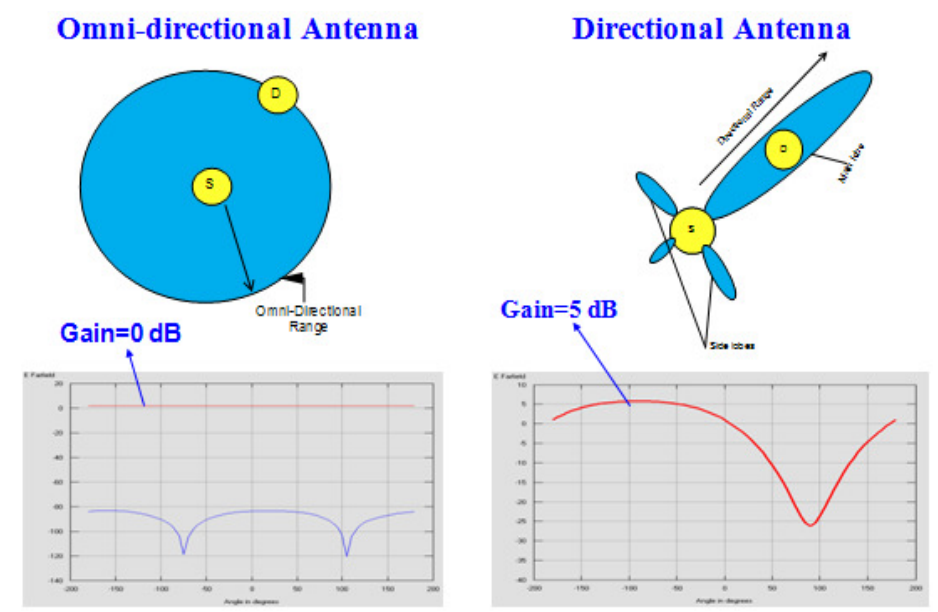

Figure 3. Radiation Patterns of Omnidirectional and Directional Antennas

\section{Energy Saving Techniques at Routing Layer in MANETs}

Energy efficient routing protocols can be mainly categorised into two types. One category selects the path that has maximum total energy, while the other category of protocols selects the paths with nodes having residual energy greater than energy required to transfer data.

The protocols that select the path with maximum energy ensure that when a packet is transmitted from source to destination, there is enough energy available for the exchange of data. While the other category of protocols which selects the path with nodes having residual energy greater than energy required to transfer data and ensures that each node has enough energy on the selected path. However, if the path selected does not minimize the energy needed to transmit a packet from its source to its destination, the network lifetime may not be maximized. Therefore, the usage of directional antenna helps in reducing the number of hops to conserve the battery energy and in turn helps in maximizing the network lifetime.

\section{Related Work ON Directional ANTENNA AND MUltipath ROUTING IN MANETS}

Most of the papers available in literature propose MAC layer or network layer protocols for adhoc networks, using directional antenna. Su Yi et.al [1] provide a good insight on the improvement in capacity of wireless network with the usage of directional antenna. This paper also provides results for throughput improvement with combination of omnidirectional and directional antennas in Transmit (Source) and Receive (Destination) nodes. In [2] Ram Ramnathan provides a good insight on beamforming antennas, and their use in ad-hoc networks. This paper also provides a rough comparison of relative lesser interference potential with: only beamforming, only power control and when both beamforming and power control are used together. The research in $[3,4,5]$ suggests the use of directional antennas for increasing the throughput and enhancing the performance of wireless networks.

However, the above cited research papers do not address a detailed formulation to link the relative improvement in Received Signal Strength (RSS), range and reduction in transmit power when high gain or moderate gain directional antenna replaces the conventional low gain omnidirectional antenna in a MANET system. This paper illustrates the potential of directional antenna to ensure the performance improvement of the communication link relative to 
International Journal of Wireless \& Mobile Networks (IJWMN) Vol. 4, No. 4, August 2012

omnidirectional antenna. Further, with the directional antennas establishing the link, it is all the more necessary to ensure that the direction of beam (peak gain) of the antenna of the transmit node is aligned with the direction of peak gain of the antenna of the receive node. In case of mismatch in antenna beam pointing angle between the two communicating nodes, there shall be a consequent degradation in the performance of communication link. This paper provides a quantitative analysis of the degradation in performance of the communication link in the presence of misalignment (angular error) between the beam pointing angles of transmit and receive nodes. An estimate of the required increase in the transmit power of the link to avoid the performance degradation and to maintain link quality as with ideal zero beam pointing error case is also provided.

The initial design and evaluation of two techniques for routing improvement using directional antennas in mobile ad hoc networks are discussed in [6]. In the first step directional antenna is used to transmit selected packets over a longer distance. Relatively more number of packets is transmitted over a shorter distance. The adaptive combination of two is called as bridging permanent network partitions. In a subsequent step, network without permanent partitions, directional antenna is used to repair routes in use, by using the capability of directional antenna to transmit packets over longer distance. In [7] the authors have proposed an energy-efficient, distributed, scalable and localized multipath search algorithm to discover multiple node-disjoint paths between the sink and source nodes for wireless sensor networks. A reactive source routing protocol referred to as Multipath Directional Antenna ad-hoc Routing (MDAR) is proposed in [8]. A distinctive feature of MDAR is that the routing table records multiple choices of routes to each destination, so that when one route encounters busy channel, an alternative route can be selected immediately.

\section{Analysis and relative comparison of Link Performance With DIRECTIONAL AND OMNIDIRECTIONAL ANTENNAS}

From the joint perspectives of antenna engineering and its relevance to system performance of the links established through participating nodes, the communication range, RSS, Transmitter power to maintain the link performance and resource utilisation particularly with respect to Battery power emerge as more critical parameters. This section provides extensive analysis of the enhancement of the parameters listed above with the use of directional antenna when compared to that of omnidirectional antenna.

\subsection{Improvement in Range due to Gain of Directional Antenna}

Friis transmission formula is used in wireless communication to calculate the power received by the antenna at the receiver section under idealized conditions. Friis transmission formula is defined through

$P_{r}=P_{t} \times G_{t} \times G_{r} \times\left(\frac{\lambda}{4 \pi R}\right)^{2}$

Where, $P_{t}, P_{r}, G_{t}, G_{r}, R$ and $\lambda$ represent Transmit Power, Receiver Power, Transmit antenna gain, Receive antenna gain, Range and Wavelength respectively.

Let, $R_{\text {omni }}, R S S_{\text {omni }}, P_{\text {Tomni }}, \lambda, G_{\text {Tomni }}$ and $G_{\text {Romni }}$ represent Range of Omnidirectional antenna, Transmit power with omnidirectional antenna, Wavelength in meters, Gain of Transmit omnidirectional antenna and Gain of receive omnidirectional antenna respectively. 
Then we have,

$$
\begin{aligned}
& \operatorname{RSS}_{\text {cmni }}-P_{\text {Tomni }} \times G_{\text {Tomni }} \times G_{R \text { omni }} \times \frac{\lambda^{2}}{4^{2} \pi^{2} R_{\text {onni }}{ }^{2}} \\
& R_{\text {omni }}^{2}=P_{\text {Tomni }} \times G_{\text {Tomni }} \times G_{R \text { omni }} \times \frac{\lambda^{2}}{4^{2} \pi^{2} R S S_{\text {omni }}} \\
& R_{\text {omni }}=\frac{\lambda}{4 \pi} \sqrt{\frac{P_{T \text { omni }} G_{T \text { ammi }} G_{R \text { omni }}}{R S S_{\text {omni }}}}
\end{aligned}
$$

The increase in the communication range $\Delta R$ due to directional antenna can be related to the term directional gain ratio $g_{r}$, where $g_{r}=G_{D A} / G_{O M N I}$

$\Delta R=R_{r m i n}\left(g_{m}-1\right)$

Effective range $\mathrm{R}_{\text {effective }}=\mathrm{R}_{\text {omni }}+\Delta \mathrm{R}$

In Equation (3), $\boldsymbol{g}_{r}$ involves $\mathrm{G}_{\mathrm{DA}}$ and $\mathrm{G}_{\mathrm{omni}}$ where,

$\mathrm{G}_{\mathrm{DA}}=$ Gain of Directional Antenna $\left(\mathrm{G}_{\mathrm{DA}}=\mathrm{G}_{\mathrm{TDA}}=\mathrm{G}_{\mathrm{RDA}}\right)$

$\mathrm{G}_{\mathrm{omni}}=$ Gain of Omnidirectional Antenna $\left(\mathrm{G}_{\mathrm{omni}}=\mathrm{G}_{\mathrm{T} \text { omni }}=\mathrm{G}_{\mathrm{R} \text { omni }}\right)$

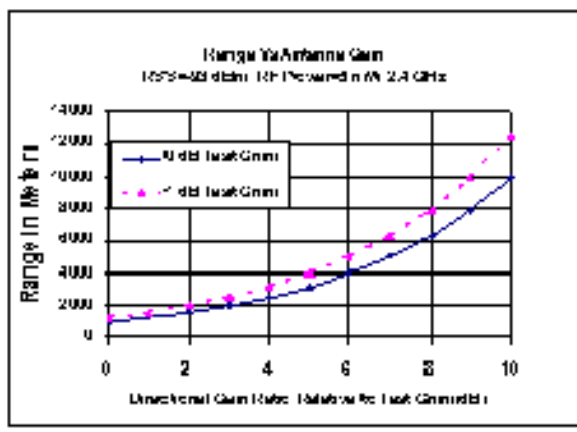

(a)

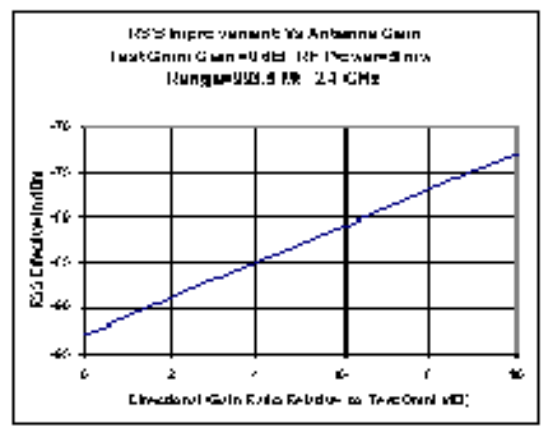

(b)

Figure 4. Influence of Directional Antenna Gain on Communication Range and Improvement of RSS effective with Directional Gain

Figure 4(a) illustrates the improvement in the communication range solely due to the gain of the directional antenna relative to omnidirectional antenna keeping the transmit power $\mathrm{P}_{\mathrm{T}}=5 \mathrm{~mW}$ and $\mathrm{RSS}=-93 \mathrm{dBm}$ to be the same in both cases of directional as well as omnidirectional antennas with the link operating at $2.4 \mathrm{GHz}$. The profile of increased gain variation exhibits much sharper feature than an exponential function.

\subsection{Improvement in Received Signal Strength (RSS) due to Directional Antenna}

If the parameter $P_{r}$ in Equation 1 is considered as $\operatorname{RSS}_{\text {omni }}$, with $G_{T o m n i}$ and $G_{R o m n i}$ as omnidirectional antenna gain, then we have, 
International Journal of Wireless \& Mobile Networks (IJWMN) Vol. 4, No. 4, August 2012

$R S S_{\text {omni }}=P_{\text {Tamni }} \times G_{\text {Tomni }} \times G_{R \text { omni }} \times\left(\begin{array}{c}\lambda \\ 4 \pi R_{\text {orni }}\end{array}\right)^{2}$

The improvement in the RSS with the use of directional antenna can be written as,

$\Delta_{R S S}=R S S_{\text {ommi }}\left[\left(g_{r}\right)^{2}-1\right]$

The effective RSS at the receiver of the link with the directional antennas replacing the omnidirectional antennas is

$R S S_{\text {offoctive }}=R S S_{\text {ommi }}+\Delta_{\text {RSS }}$

Figure 4(b) depicts the variation of realizable improved $\operatorname{RSS}_{\text {effective }}$ as a function of the directional gain ratio. Apart from linear nature of variation, the result depicted in Figure 4(b) also reveals that for a $\mathrm{dB}$ increase in gain ratio, the corresponding improvement in $\mathrm{RSS}_{\text {effective }}$ is $2 \mathrm{dBm}$.

In this section, we show the reduction in required transmission power with the use of directional antenna, when compared to that required for omnidirectional antenna. Based on the Friis Transmission formula given by Equation 1, we have,

$\Gamma_{T \text { omni }}=\begin{aligned} & R S S_{\text {omni }} 4^{2} \pi^{2} R_{\text {omni }}^{2} \\ & G_{\text {Tomni }} G_{R \text { omni }} \lambda^{2}\end{aligned}$

The reduction in the Transmitter power $\Delta \mathrm{P}_{\mathrm{T}}$ because of higher gain of directional antenna can be expressed as

$\Delta P_{T}=P_{\text {Tomin }}\left[1-\left(1 / g_{r}\right)^{2}\right]$

The definition of Gain Ratio remains the same as in earlier sections. The actual transmit power required by the directional antenna to retain the range and $\mathrm{RSS}_{\text {omni }}$ of the omnidirectional antenna is given by

$P_{\text {Teffotive }}=P_{\text {Tomn: }}-\Delta P_{T}$

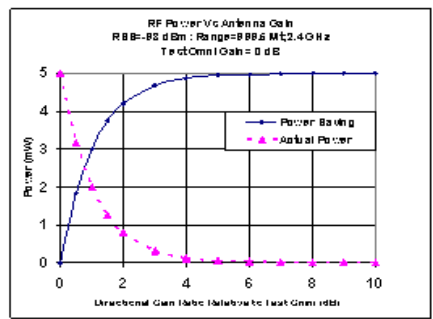

(a)

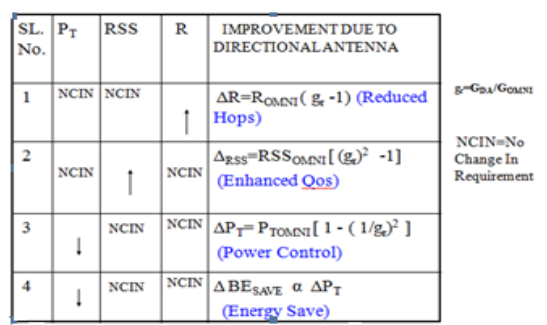

(b)

Figure 5. Reduction in RF Transmitter Power with Directional Gain and Multifaceted Advantages of Directional Antenna 
Figure 5(a) shows the reduction in the RF Transmitter power with the use of directional antenna keeping the RSS as well as range parameters the same in both the directional and omnidirectional cases. Drastic reduction in transmitter power is evident with smaller initial increase in directional gain and then exhibiting asymptotic nature with higher directional gain.

\subsection{Multidimensional Versatility of Directional Antenna}

Figure. 5(b) summarizes the highlights the advantages of using directional antenna. In Figure. $5(\mathrm{~b}), \Delta \mathrm{BE}_{\text {Save }}$ denotes the reduction in the energy of battery with the directional antenna to retain or maintain the link performance as in the case of omnidirectional antenna.

With increased communication range $(\mathrm{R})$, the number of hops required for data exchange in an ad-hoc network can be reduced thus reducing the end-to-end latency. Higher RSS increases the signal quality at the receiver, thus providing enhancement in Quality of Service (QoS). With the reduction in transmitter power, the battery energy consumed at each node decreases, thus providing longer battery life, which further increases the network lifetime. Power control also reduces interference among neighboring nodes thus increasing the efficiency of the network.

\subsection{Estimation of Battery Energy for Given Data Transmission}

Research publications $[9,10,11,12]$ address the issue of need and challenges in the realization of energy efficient ad-hoc/MANET. However, a formulation or a detailed formulation with clearly illustrated steps to compute the battery energy required for a specified data transmission seems to have not been addressed in the literature even though this is very significant from system design perspective. This section summarizes the detailed steps through which one could compute the required battery energy and the formulation shall take into account the data pertaining to the power efficiency of the associated transceiver design as well as the specified link performance parameters.

From the classical relationship between Power, Energy and Time, one can draw analogy to relate the requirement of Battery Power $\left(\mathrm{P}_{\mathrm{Bat}}\right)$ and Battery Energy $\left(\mathrm{BE}_{\mathrm{Data}}\right)$ for transmission of given data.

$$
\mathrm{BE}_{\text {Data }}=\mathrm{P}_{\mathrm{Bat}} \mathrm{XT}_{\mathrm{ON}}
$$

Where $\mathrm{T}_{\mathrm{ON}}=$ Transmitter ON Time.

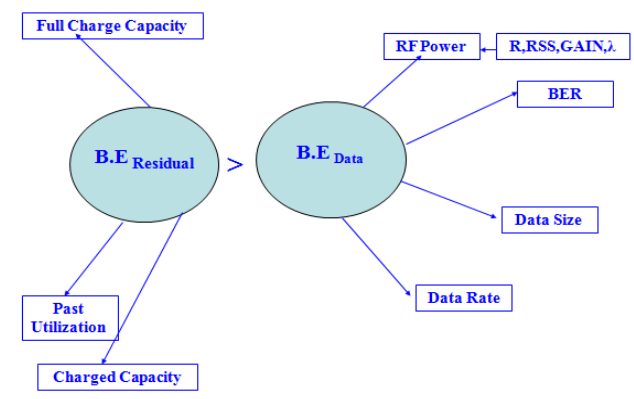

Figure 6. Requirement of Battery Energy for Data Transmission

$\mathrm{T}_{\mathrm{ON}}$ is dependent on the size of data, data rate as well as channel condition between the Transmit and Receive ends of the link. At an instant of time for data transmission, the following inequality should be satisfied. 


$$
\mathrm{BE}_{\text {Residual }}>\mathrm{BE}_{\text {Data }}
$$

Where, $\mathrm{BE}_{\text {Residual }}$ denotes the available Battery Energy at that instant for operation. Many research publications dwell with energy efficient routing protocols in ad-hoc/MANET. However, it is difficult to find a formulation to estimate or compute $\mathrm{BE}_{\text {Data }}$ that has a generic or empirical appeal and which facilitates a better appreciation from system design considerations. In this section, a generic procedure is outlined to systematically compute the $\mathrm{BE}_{\text {Data. }}$ As illustrated in Figure 6, the Battery Energy is dependent on communication range (R), RF power of transmitter, gain of the transmitting as well as receiving antennas, the expected or threshold RSS at the receive node and wavelength.

For a specified range, RSS, Gain of Transmit and Receive antennas and wavelength, the RF Transmit Power can be computed using equation (11):

$$
P_{T}=\frac{R S S 4^{2} \pi^{2} R^{2}}{G_{T} G_{R} \lambda^{2}}
$$

The relationship between $\mathrm{P}_{\mathrm{T}}$ and Battery Power $\mathrm{P}_{\mathrm{Bat}}$ is very specific to the RF design of the Transceiver module of the ad-hoc/MANETs as well as the type of battery used in it. However, the data sheet of RF transceiver usually provides the relationship between $\mathrm{P}_{\mathrm{T}}$ and $\mathrm{P}_{\mathrm{Bat}}$ in tabular form. Either a look up table or an algebraic expression deduced from the tabulated data is required to estimate the $\mathrm{P}_{\mathrm{Bat}}$ for a specific PT determined through Equation (11). Such recourse of computing the Battery Power $\mathrm{P}_{\mathrm{Bat}}$ shall further facilitate built in provision for computation of Battery Energy $\mathrm{BE}_{\text {Data }}$ through Equation (9). Figure 7 illustrates the least square curve fitting equation obtained to relate the RF Power $\mathrm{P}_{\mathrm{T}}$ and Battery Power $\mathrm{P}_{\mathrm{B} a t}$ for a typical RF transceiver widely used in $2.4 \mathrm{GHz}$ band. Satisfactory correlation between the results of derived functional relation and the reference data is noticed in Figure 7.

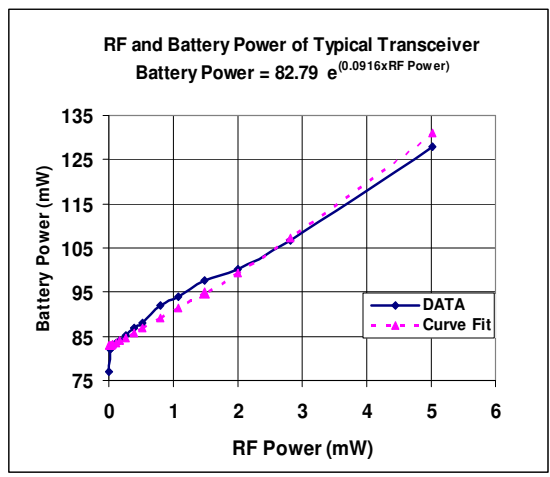

Figure 7. Derivation of Functional Relation between RF and Battery Power

\subsection{Effect of Beam Pointing Angle Error of Directional Antenna on Network Performance}

In order to retain all the network performance enhancements highlighted thus for, one has to also address the importance of alignment of direction of peak gain of the transmitting beam of the source node with the corresponding receiving beam of the destination node. Misalignment of the beam peaks of the source and destination nodes result in a parameter defined as a Beam Pointing Angle (BPA) Error or Beam Pointing Error (BPE) as shown in Figure. 8. In many of the aperture antennas with moderate gain, the shape of the radiation pattern up to $-10 \mathrm{~dB}$ points is assumed to follow functional distribution $e^{-p \theta^{\mathbb{2}}}$. 


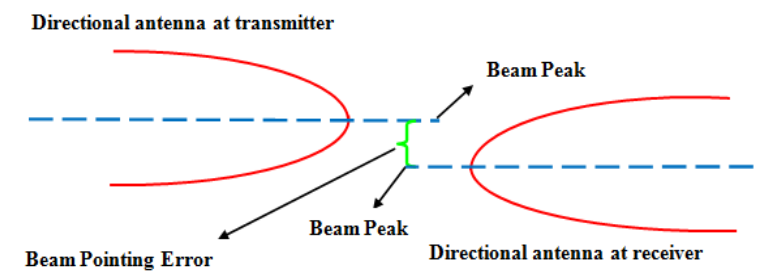

Figure 8. Illustration of Beam Pointing Angle Error of Directional Antenna

The value of the constant 'p' can be derived with the plot of the radiation pattern at say $-3 \mathrm{~dB}$ or $-10 \mathrm{~dB}$ points relative to the beam maximum angle. This procedure has been adopted to analyse the effect of BPA or BPE on the link performance metrics such as range and the RF power requirement as well as Battery Power. The degradation in the range performance as a function of BPA is shown in Figure 9(a). As expected, the degradation becomes more pronounced with increase in BPA.

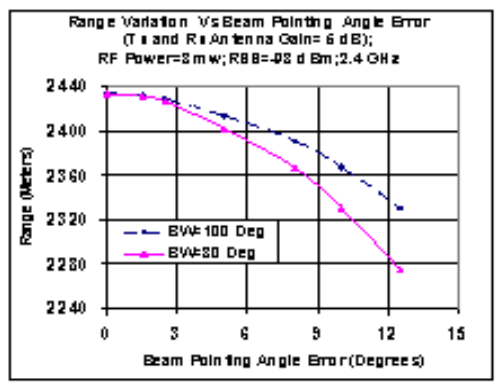

(a)

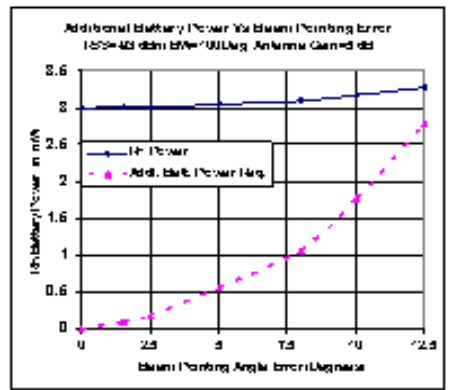

(b)

Figure 9. Influence of Beam Pointing Angle Error on Network Performance and Effect of Beam Pointing Angle Error on RF and Battery Power

If one desires to regain the ideal link performance despite the presence of BPA or BPE, it can be realized only at the expense of increased RF transmit power. This in turn would result in additional consumption of battery power resulting in reduced operational time of the link. Figure 9(b) explains the undesirable influence of BPA on the link transmitter power and which in turn has a negative effect on the required battery power to sustain the link performance.

\section{Energy Efficient Multi Path Routing Protocol}

The Energy Efficient Protocol proposed in this paper lists all possible paths from source to the destination with no repetition and avoiding loop formation in path computation. Once the multiple paths are computed, next step is to decide which among them is the best from energy efficiency consideration. To arrive at that decision, the protocol ranks each path based on Hop count, battery energy required to send the data and residue of residual energy of each path. From among all the multiple paths, the path with highest weightage which corresponds to the most energy efficient is selected.

\subsection{Hop Count Minimization}

Once the most energy efficient path is chosen based on Decision Weights, further optimization of the number of hops is the next step. Ideal requirement is that the data transfer be accomplished with a single hop irrespective of the distance between the source and the 
International Journal of Wireless \& Mobile Networks (IJWMN) Vol. 4, No. 4, August 2012

destination nodes. However, the power handling capacity of node, gain of the antenna associated, desired RSS at the Receiver and Wavelength determine the maximum communication range that is realizable and is calculated using the formula

$$
\max \operatorname{Dir}=\left[\frac{\lambda}{4 \pi}\right] \sqrt{\frac{P_{T D i r} \times G_{T D i r} \times G_{R D i r}}{R S S_{D i r}}}
$$

For $\mathrm{G}_{\mathrm{TDir}}=\mathrm{G}_{\mathrm{RDir}}=5 \mathrm{~dB}, \mathrm{P}_{\mathrm{Tdir}}=5 \mathrm{~mW}, \mathrm{RSS}_{\text {Dir }}=-93 \mathrm{dBm}$ and $\lambda=0.125 \mathrm{~m}$, the maximum range is $\max$ Dir=3141.87 $\mathrm{m}$. The disadvantage of selected optimized path when the size of the network is large is that the distance between nodes is also very large. It is also associated with a relatively higher number of hop counts which in turn may not ensure error free communication. To overcome this, Proposed Protocol has a feature of hop count minimization using maximum range of directional antenna. Using directional max distance as a parameter, the protocol searches the possible minimum number of hops required to cover the already chosen energy efficient multipath.

From Figure 10, it can be seen that initial path selected by the protocol has 9 hops between yjr source node 11 and destination node 24 . Using directional max range that is $3141.87 \mathrm{~m}$, the distance between source and the destination nodes is considered to check whether it is less than directional 'max Dir', so that possibility of one hop communication can be ascertained. But in the scenario under consideration, the distance between the source-destination nodes is $3824.3 \mathrm{~m}$ which is greater than directional max range implying the non-feasibility of one hop communication (Figure 10).

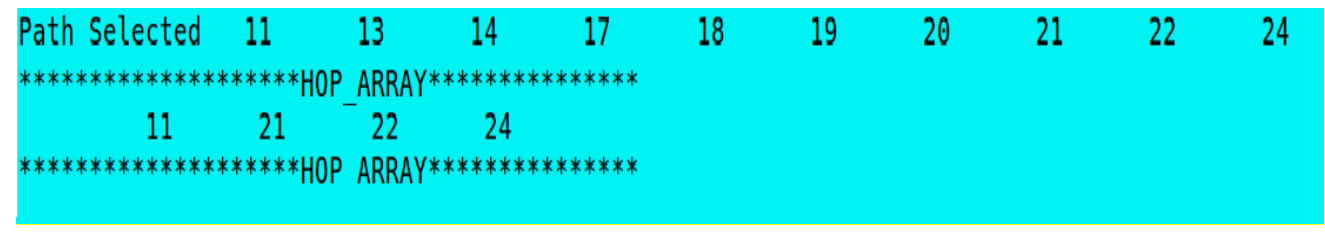

Figure 10. Multi Hop Communication between Source Node 11 and Destination Node 24

If one hop communication between source and destination nodes is not possible, then the distance between source node 11 and the node 22 which is next closest node to the destination node and also furthest from the source node next only to the destination node is checked. The range between the source node 11 and the node 22 is $3259.6 \mathrm{~m}$ which is still higher than the directional max range. Recursively, the next node from the destination end that is farthest to the source node is chosen and the range between these is checked. As shown in Figure 11, distance between source 11 and node 21 is verified and its value of $3000 \mathrm{~m}$ is less than directional max range. This means that node 21 is within one hop communication range of directional antenna of source 11. So all the nodes in the chosen energy efficient multipath between source node 11 and node 21 are skipped and the final hop count optimized path is of 3 hops and this is elucidated from Figure 11. In the chosen scenario, the number of hops of the selected path without refined hop count optimization is 9 which has been reduced to 3 hops which ensures error free and energy efficient communication when compared to communication through selected path. 
International Journal of Wireless \& Mobile Networks (IJWMN) Vol. 4, No. 4, August 2012

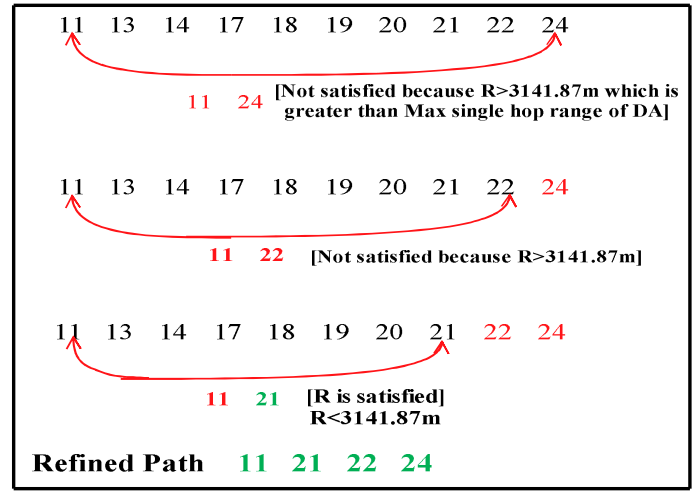

Figure 11. Pictorial Representation of Hop Count Minimization

\subsection{Energy Conserved Using Directional Antenna Hop Count Minimization}

Hop count minimization feature of the proposed protocol is very useful when the network size is large. From Table 1, it is seen that energy utilized by selected multiple path with 9 hops is 94.83 Joules where as the energy used by hop count optimized path with 3 hops is 36.72 Joules. In these computations, the data size of $5 \mathrm{MB}$ and data rate of $40 \mathrm{kbps}$ have bee assumed. Hence the energy save is 58.11 Joules which amounts to $62.11 \%$. Thus the stated principal theme of development and demonstration of energy efficient multi path routing protocol using directional antenna for MANET featured with reduction of number of hop counts potentially assures not only energy saving but also an error free communication.

Table 1. Energy Utilization and Conservation between Two Paths

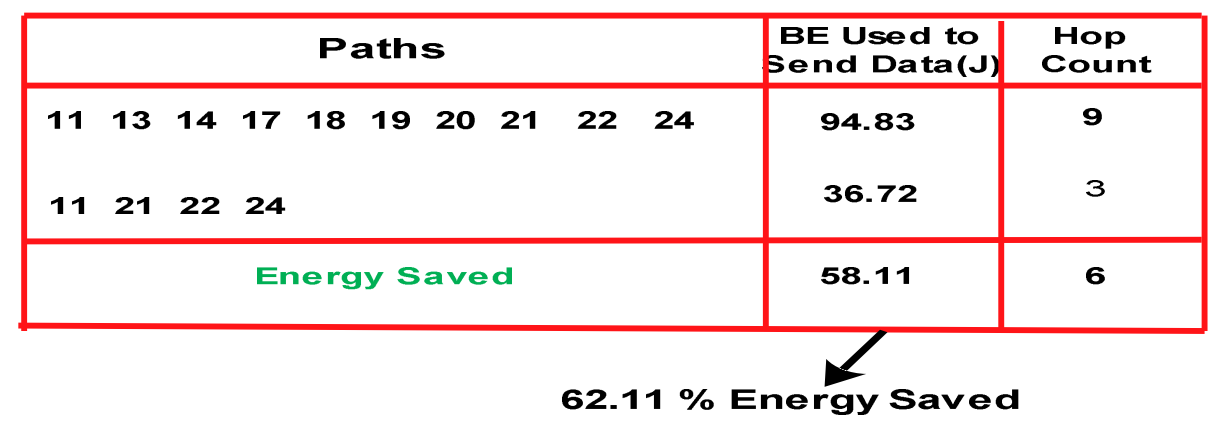

\section{Results}

Figure 12 displays the QualNet simulation showing the placement of 25 nodes in the network(Give some additional details). 
International Journal of Wireless \& Mobile Networks (IJWMN) Vol. 4, No. 4, August 2012

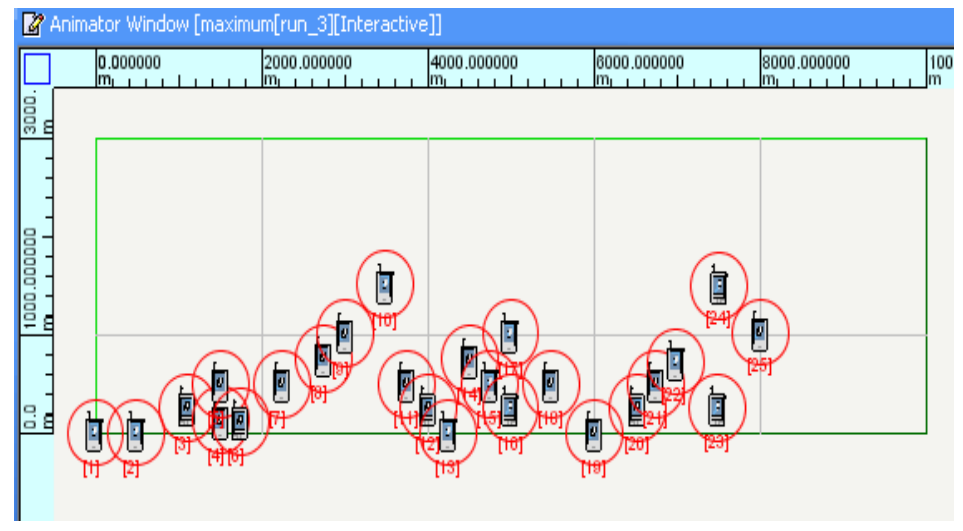

Figure 12. QualNet Animator (B)

\subsection{Performance Analysis}

\section{A. Results of Packet Delivery Ratio Results of Scenario with 25 nodes}

Figure 13 shows the error free data transmission from the source node 11 to destination node 24 using the refined final path following the hop count minimization feature of the proposed protocol.
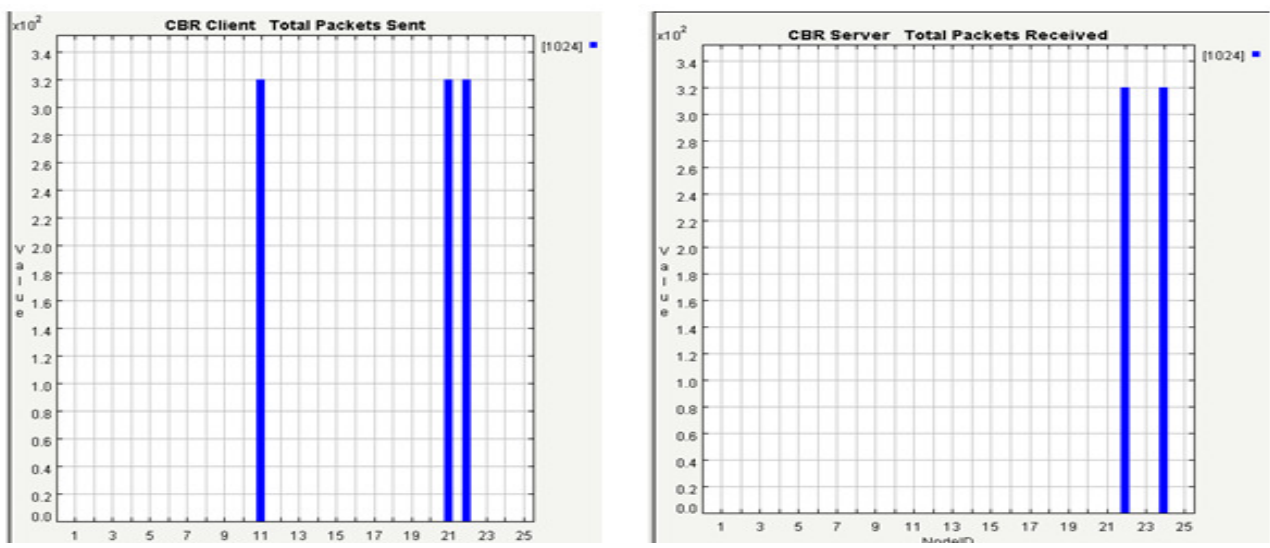

Figure 13. Packet Sent and Received

When the path for data communication contains more number of hops, usually error free communication is not expected/ensured. The combination of directional antenna, Energy Efficient multipath selection and further refinement of the selected path with optimization of hop counts has resulted not only an enormous saving of energy as shown Table 1 but also an error free transmission,

\section{2 Analysis of Throughput}

Figure 12 is a typical depiction of relatively larger size of network of 25 nodes. Throughput is an important performance metric of any wireless network. As can be seen from the results of Figure 14, the total number of 79 Kilo Bytes transmitted by the sender node 11 has been received by the first intermediary nodes 21 . 

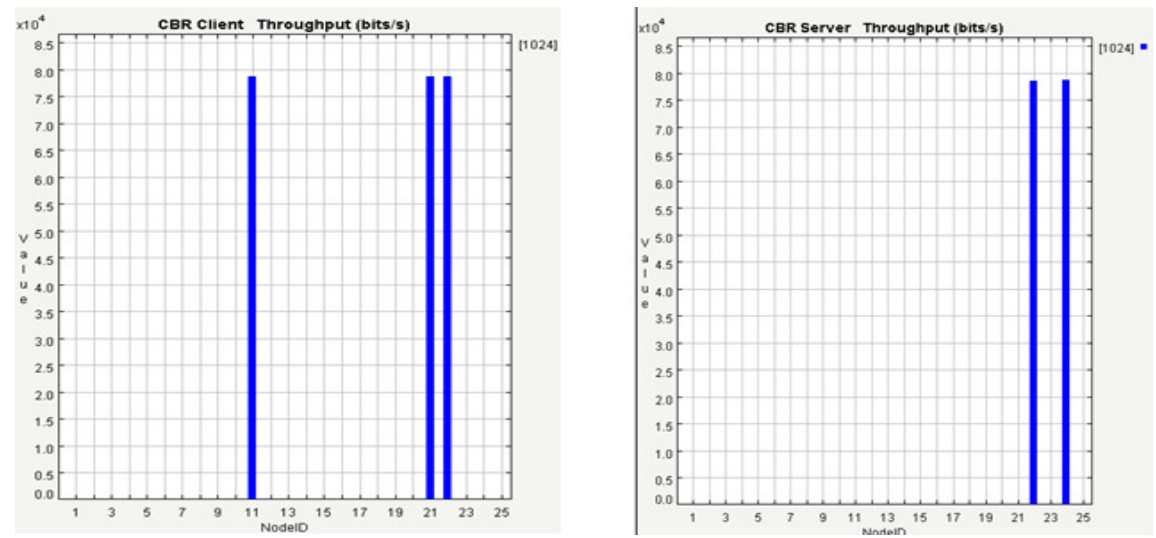

Figure 14. Throughput

Subsequently the transmission of it by node 21 has been received by the second intermediary node 22 also and ultimately culminating in the reception of all the 79 Kilobytes by the destination node 24 . Figure 14 amply demonstrates the effectiveness of the energy efficient multipath routing protocol using directional antenna proposed in this paper.

\subsection{Performance Analysis of End to End Delay}

\section{A. Scenario with 25 Nodes (9 Hops)}

The delay (elapsed time between the transmission and reception at the successive nodes) strongly depends on the distance between the transmit and receive nodes. The time elapsed between the communication starting with the transmission at the source node and the reception at the destination node is the algebraic summation of the delays caused at each hop along the selected multi path. The Figure 15 displays the amount of time elapsed between the transmit and reception processes at the successive nodes along the selected path comprising 9 hops. The end to end delay encountered by the individual hops varies from a minimum of $5 \mathrm{~ms}$ to a maximum of $8.6 \mathrm{~ms}$.

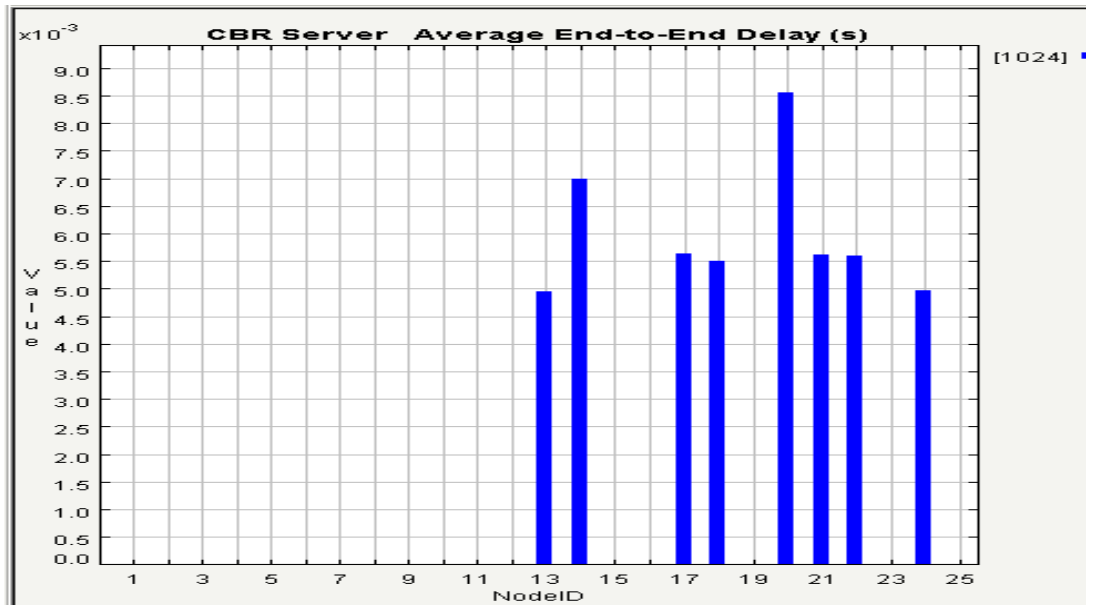

Figure 15. End To End Delay of Selected Path 


\section{B. Scenario with 25 Nodes (3 Hops)}

The Figure 16 intends to illustrate the advantage of the hop count optimization feature of the proposed protocol. While the results of Figure 15 pertain to the energy efficient multipath chosen by the decision weight of the analysis for 25 node scenario with 9 hops, the Figure 16 refers to the same scenario but with the number hops being reduced to 3 . The results of Figure 16 spring no surprises since it is on expected lines in the sense that smaller the hop counts, lesser the spread in the variation of end to end delay. A nearly uniform and a relatively lesser end to end delay of about $3.4 \mathrm{~s}$ is observed along the refined energy efficient multipath.

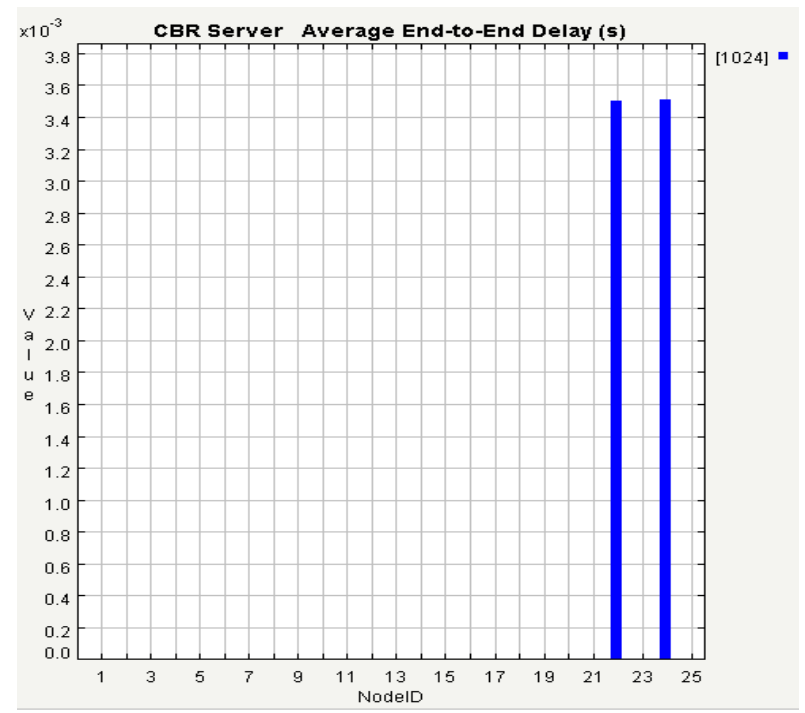

Figure 16. End To End Delay of Hop Count Optimized Path

\subsection{Performance Analysis of Jitter}

\section{A. Scenario with 25 Nodes (9 Hops)}

Jitter is also one of the parameters to be considered in the performance evaluation of a Wireless Communication system such as MANET. It is defined as variation in time between the packets arriving at the destination. The variation can be attributed to one or more of the followings; Network Congestion, end to end to delay or changes in the route. 
International Journal of Wireless \& Mobile Networks (IJWMN) Vol. 4, No. 4, August 2012

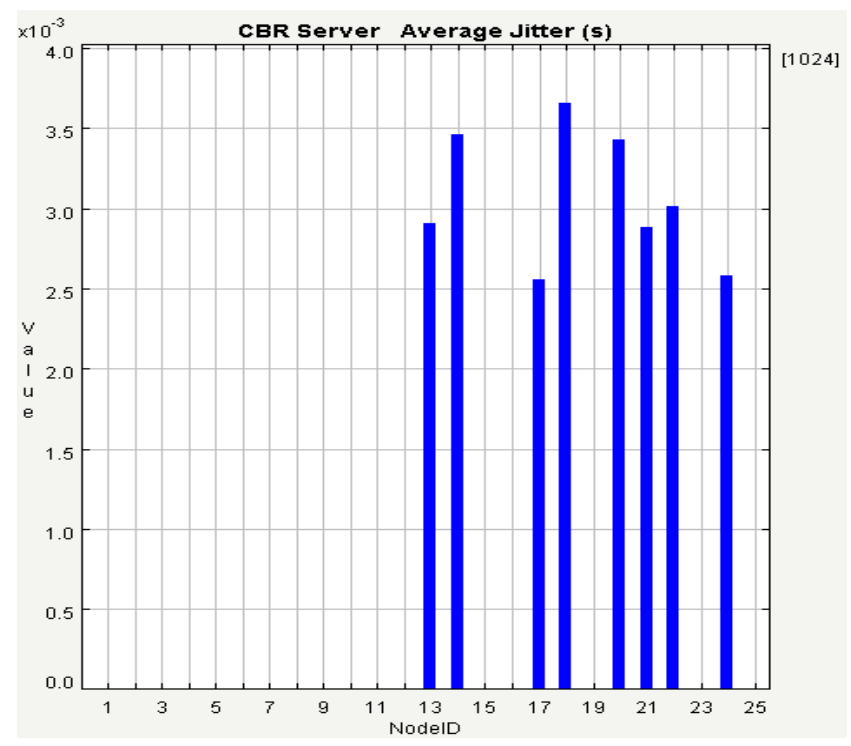

Figure 17. Jitter of Selected Path

The Figure 17 shows packets delay is more in the selected path due to the varying positions of the nodes and more number of hops along the path. Higher jitter can seriously affect the quality of data or streaming audio and/or video.

\section{B. Scenario with 25 Nodes (3 Hops)}

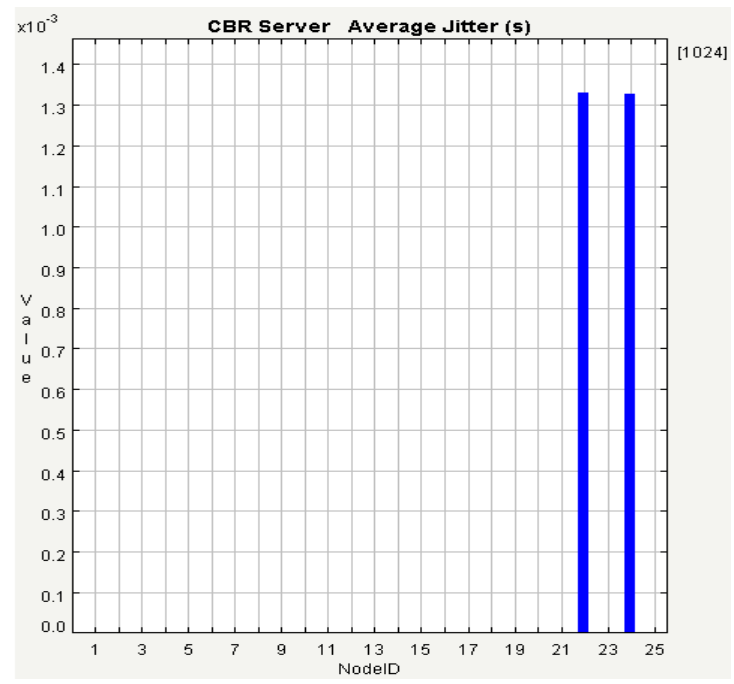

Figure 18. Jitter of Hop Count Optimized Path

When the selected path in Figure 17 is further optimized with hop count feature of developed protocol, jitter is considerably low as shown in Figure 18.

\section{CONCLUSiON}

The paper proposes the development of an energy efficient multipath routing protocol with directional antenna for MANET as an optimization task as well as a multidisciplinary entity encompassing Antenna Engineering, Microwave Link Budget to treat intermediate wireless 
International Journal of Wireless \& Mobile Networks (IJWMN) Vol. 4, No. 4, August 2012

links, Algorithms to determine the most efficient path for reliable connectivity between the source and destination nodes, comprehensive relation between the RF power and the pertinent Battery Power of the transmit nodes involving conventional electrical power engineering basics as well as choice and optimization of Route metrics to arrive at decision logic for the path selection. This paper presents a comprehensive analysis to link all the multi-disciplinary view points involved in the development of desired multipath routing protocol with requisite technical details. Through the implementation of RF power control, the paper emphasizes and elucidates the importance of Directional antenna in MANET to conserve battery energy and still retaining all other desirable system performance attributes.

Through numerical simulations, the multi-dimensional desirable performance attributes of wireless link such as improved range, improved RSS, reduced RF transmit power and consequent reduced consumption of battery power have been analyzed. Majority of the formulations on Antennas presented in this paper stem from the Friis transmission formula which is well known for its simplicity, importance and empirical nature, that is essential for the design of wireless link Undesirable effect of Beam Pointing Angle error of directional antenna leading to degraded range performance has been illustrated through a typical case study Simulations confirm that significant improvement in energy saving is a reality if the:Range (distance) between the source and the destination nodes is well beyond the maximum directional range of the network operating with its rated peak RF power and Directional Gain is couple of dB higher than Omni Gain. Simulation results on range enhancement with directional antenna reveal that the profile of increased range with variation in antenna gain exhibits an exponential behaviour and can be very effective in the reduction of number of hops Results also confirm that a node with directional antenna requires considerably much lower RF power than that required with an omni directional antenna to retain the same link performance and this in turn leads to smaller battery requirement resulting in energy saving Through typical case studies, the simulation results amply confirm the significant improvement in the life time of the network by using directional antennas.

Simulation results of the implemented multipath Routing protocol associated with built in hop count reduction feature reveal energy saving of $62.11 \%$ for a typical MANET scenario of 25 nodes, $5 \mathrm{MB}$ data and data rate of $40 \mathrm{Kbps}$. From a system perspective, performance of the proposed routing technique can be justified as Highly Energy Efficient Multipath Routing Protocol for MANETs. Contributions that can be derived out of this paper will be of potential utility to address the realistic design challenges and issues pertaining to wireless network in general and energy efficient MANETs in particular. Emphasis on requisite analysis and details on multi-disciplinary aspects of Antennas, Power Computations, algorithm for multi-path determination and Pre Processor to access simulator is a first step in a frame work for development of Network system simulator.

\section{REFERENCES}

[1] Su Yi, Yong Pei and Shivkumar Kalyanaraman,(2003) "On the Capacity Improvement of Ad Hoc Wireless Networks using Directional Antennas”, Mobihoc, pp. 108-116.

[2] R. Ramnathan, (2001) "On the Performance of Ad Hoc Networks with Beamforming Antennas", Mobihoc, pp. 95-105

[3] A. Nasipuri, K. Li and U. R. Sappidi, (2003) "Power Consumption and Throughput in Mobile Ad Hoc Networks using Directional Antennas”, IEEE International Conference on Computer, Communication and Networking

[4] A. Spyropoulos and C.S. Raghavendra, (2002) "Energy Efficient Communications in Ad Hoc Networks Using Directional Antennas”, INFOCOM’02. 
International Journal of Wireless \& Mobile Networks (IJWMN) Vol. 4, No. 4, August 2012

[5] A. Arora, M. Krunz, and A.Muqattash, (2004) "Directional Medium Access Protocol (DMAP) with Power Control for Wireless Ad Hoc Networks", Global Telecommunication Conference

[6] Amith, K. S., and David, B. (2005) "Routing Improvement Using Directional antennas in MANETs", IEEE International Conference on Mobile Ad hoc Networks, Fort Lauderdale, Florida, 304-313

[7] Luz, Y. M., and Vincent Wong W. S. V. (2007) “An Energy-Efficient Multipath Routing Protocol for Wireless Networks",. International Journal of Communication System 20(7), pp. 747-766

[8] Guoqing Li, L. Lily Yang, W. Steven Conner, Bahareh Sadeghi (2003) "Opportunities and Challenges for Mesh Networks Using Directional Antennas”.. Intel Corporation, Communication Technology.

[9] Bashir Yahya and Jalel Ben-Othman,(2009) "Robust and Energy Efficient Multipath Routing Protocol for Wireless Networks”, Global Telecommunication Conference, pp. 1-7.

[10] R. Vidhyapriya and P.T. Vanathi, (2006) "Energy Efficient Adaptive Multipath Routing for Wireless Sensor Networks”, International Journal of Computer Science, pp. 1-9.

[11] Chansu Yu, Ben Lee and Hee Yong Youn, (2003) "Energy Efficient Routing Protocols for mobile ad hoc Networks", Wireless Communications and Mobile Computing, pp. 959-973.

[12] Busola S. Olagbegi and Natarajan Meghanathan, (2010) "A Review of Energy Efficient and Secure Multicast Routing Protocols for Mobile Ad Hoc Networks", International Journal on applications of graph theory in wireless ad hoc networks and sensor networks.

\section{Authors}

Sandhya Chilkuri obtained Bachelors Degree in Computer Applications from Kuvempu University, India. She graduated with a degree of M.Sc (Engineering) in Computer Science and Networking from Coventry University, UK at M.S.Ramaiah School of Advanced Studies (MSRSAS), Bangalore, India. Currently Sandhya is a research scholar at MSRSAS pursuing her Ph.D. degree. Her field of research comprises simulation and modelling of Mobile Ad-Hoc Network, Multipath Routing Protocols and applications of Antenna Engineering in the design of Energy Efficient Ad Hoc network.

Rinki Sharma is working as Assistant Professor in M.S.Ramaiah School of Advanced Studies. She has 7 years of experience in teaching and research. Her main areas of interest are Computer Networks, Network Programming, Wireless Networks and Protocols and Mobile Ad-hoc Networks. Presently she is pursuing her Ph.D from Coventry University U.K., in the area of Mobile Ad-hoc Networks.

Govind R Kadambi, is a Professor and Dean at M.S.Ramaiah School of Advanced Studies, Bangalore, India. He received B.E. degree in Electronics and Communications from the University of Mysore, India, and the M.S. and Ph.D. degrees from the Indian Institute of Technology (IITM), Madras. Prior to joining MSRSAS, he was associated with various R\&D Labs in India and abroad. His current research interests are mainly focused on Antennas, Computational Electromagnetics, Digital Beamforming, Mobile Ad-Hoc Networks, Signal Processing for Wireless Communication and Systems Engineering.

Deepali. R.Borade graduated with a degree of M.Sc (Engineering) in Computer Science and Networking from Coventry University, UK at M.S.Ramaiah School of Advanced Studies (MSRSAS), Bangalore, India. Her field of interest is Mobile AdHoc Network.
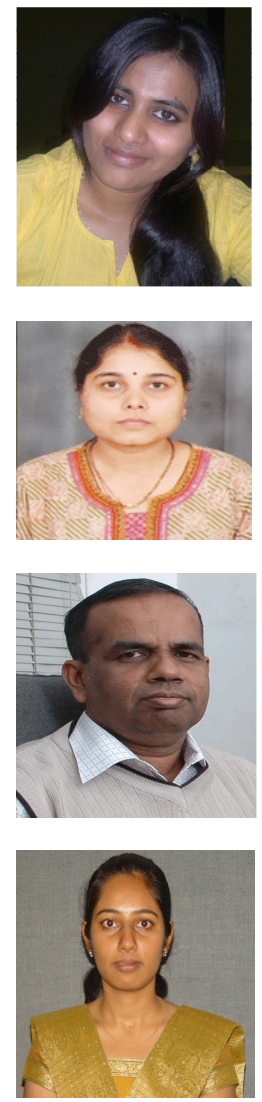